Article

\title{
Research on Improvement of the Combination Method for Conflicting Evidence Based on Historical Data
}

\author{
Shuai Yuan ${ }^{1,2}$ and Honglei Wang ${ }^{1,3, *}$ \\ 1 College of Management, Guizhou University, Guiyang 550025, China; kace68@163.com \\ 2 College of Construction Engineering, Guizhou Minzu University, Guiyang 550025, China \\ 3 Key Laboratory of Internet+ Collaborative Intelligent Manufacturing in Guizhou Province, \\ Guiyang 550025, China \\ * Correspondence: hlwang@gzu.edu.cn
}

Received: 3 February 2020; Accepted: 10 March 2020; Published: 6 May 2020

\begin{abstract}
In a multi-sensor system, due to the difference of performance of sensors and the environment in which the sensor collects evidence, evidence collected will be highly conflicting, which leads to the failure of D-S evidence theory. The current research on combination methods of conflicting evidence focuses on eliminating the problem of "Zadeh paradox" brought by conflicting evidence, but do not distinguish the evidence from different sources effectively. In this paper, the credibility of each piece of evidence to be combined is weighted based on historical data, and the modified evidence is obtained by weighted average. Then the final result is obtained by combining the modified evidence using D-S evidence theory, and the improved decision rule is used for the final decision. After the decision, the system updates and stores the historical data based on actual results. The improved decision rule can solve the problem that the system cannot make a decision when there are two or more propositions corresponding to the maximum support in the final combination result. This method satisfies commutative law and associative law, so it has the symmetry that can meet the needs of the combination of time-domain evidence. Numerical examples show that the combination method of conflict evidence based on historical data can not only solve the problem of "Zadeh paradox", but also obtain more reasonable results.
\end{abstract}

Keywords: historical data; credibility; DS evidence theory; conflicting evidence

\section{Introduction}

D-S evidence theory was proposed by Harvard mathematician A.P. Dempster in the 1960s, and his student G. Shafer introduced a trust function for further development [1], thereby forming a system theory that deals with uncertainty problems by "evidence" and "combination". D-S evidence theory has received extensive attention since its inception, and researchers have conducted research on method improvements [2-13] and practical applications [14-17] and achieved a series of research achievements. Yager [2] improved the D-S evidence theory, keeping the relative proportion of evidence unchanged, and classifying conflicts into unknown sets. Sun [3] adopted the degree of conflict between the evidence to be combined to assign credibility to the evidence and solve the problem caused by the conflict of evidence. $\mathrm{Li}$ [4] averaged and weighted the probabilities of each proposition, and added it to the D-S evidence combination formula to decrease the degree of conflict. Murphy [5] arithmetically averaged all the pieces of evidence to be combined ( $n$ total) to obtain a new evidence, and used D-S rule to combine $n-1$ times to obtain the final result, which has fast convergence speed and can handle the problem brought by conflicting evidence. Deng [6] employed the similarity between the evidence to weight the average of $n$ pieces of evidence to obtain a new piece of evidence, and used the D-S 
rule to combine $n-1$ times, which has faster convergence rate and can also resolve the problem brought by conflicting evidence. Yao $[7,8]$ proposed to adopt the "neighborhood borrowing method" to resolve the conflict of evidence, and the Delphi method to modify weights of sensors, meaning a strong dependence on the scene and limiting the scope of application of the model.

It is not difficult to see that researchers have gradually realized that there are differences in the credibility of evidence with the deepening of theoretical research. In order to meet the needs of applying D-S evidence theory to practice, evidence should be weighted before combination. In practice, evidence is collected by sensors in different environments, so the accuracy of evidence is closely related to the performance of the sensor and the environmental conditions where the sensor is located when the evidence is collected. As an electronic component, the sensor will deteriorate due to aging after a certain period of time and its performance is directly related to the operating time. As far as the interference of the environment where the sensor is located, the smaller the interference, the higher the credibility of the collected evidence, and conversely, the lower the credibility of the collected evidence. In this article, the evidence is distinguished. Bad evidence refers to evidence that is very different from the actual target due to the poor performance of the sensor or excessive environmental interference. Good evidence means the opposite. Therefore, the premise that the evidence collected by each sensor is an equally credible, on which previous scholars' research based, is obviously an idealized assumption.

Moreover, researchers adopt the criterion of "not being contrary to intuitive feeling" as a measure of the effectiveness of evidence combination, which also has limitations. The criterion of "not being contrary to intuitive feeling" has strong theoretical and practical significance, and actually means the criterion of "minority obeys majority" to evaluate the combination result. As far as the combination result is concerned, "not being contrary to intuitive feeling" is consistent with "not being contrary to the facts" under the condition that the proportion of bad evidence is relatively low and the proportion of good evidence is relatively high. However, it often happens that truth is in the hands of a few. In this case, because the proportion of bad evidence is relatively high and the proportion of good evidence is relatively low, there will be a problem that "not being contrary to intuitive feeling" but "being contrary to the facts" of the combination result. Especially when researchers calculate the distance or conflict coefficient between the evidence to be combined, which strengthened the pair of evidence with high similarity and weakened the pair of evidence with low similarity $[3,6]$, the more bad evidence, the greater the error between the combination results and the facts. This is because it will improve effectiveness and convergence speed of the method when high-accuracy evidence is in the majority, but it will increase the combination error and reduce the convergence speed when low-accuracy evidence is in the majority. In the combination of evidence, if the weight of evidence from different sources can be weighted based on the performance status and environmental conditions of the sensor in which the evidence is collected, the accuracy of the combination result will be improved, and the convergence speed will be accelerated. Given that it is difficult and costly to perform real-time monitoring of performance of the sensor and environment conditions in practice, it is particularly important to find an economical and reasonable alternative.

Under normal circumstances, the performance of the sensor and the environment conditions for collecting evidence are stable for a certain period of time, which means that the internal and external state of the sensor at a certain point in time within a certain period can approximately represent the internal and external state of that period of time, especially in the case where the frequency of evidence collection by the sensor is relatively high. Moreover, when judging the credibility of the latest evidence collected by the sensor, it is completely feasible to perform based on the accuracy of the historical data collected by the sensor in the recent past period. This is because, in the context of the current era of big data, as far as the development level of computer technology and big data technology, historical data is at least partially available, that is, the historical observation data of the sensors and the corresponding real result at the time of observation have at least part of it can be recorded, stored and queried, for example with the help of blockchain technology. Blockchain, as a new application mode of computer technology such as distributed data storage, point-to-point transmission, consensus 
mechanism, encryption algorithms and so on, is based on the characteristics of consensus mechanism, data storage, network protocols, encryption algorithms, privacy protection, smart contracts, etc., which can ensure that the data stored in the certificate is truly traceable and cannot be tampered with [18]. Therefore, this article studies the method of conflict evidence combination based on historical data.

The remainder of this paper is organized as follows. Section 2 introduces the relevant concepts of evidence theory. In Section 3, the existing representative improvement methods are given. In Section 4, the improvement method in this paper is derived and the specific implementation of the proposed algorithm is given. Section 5 analyzes numerical examples. In the last section, we conclude the paper.

\section{Theoretical Foundations}

The D-S evidence theory proposed by A.P. Dempster and G. Shafer lays the research foundation for evidence combination [1]. The related concepts involved in D-S evidence theory are briefly introduced here.

Definition 1 ([1]). Let $\Omega$ be a set of proposition $A, \Omega=\left\{\mathrm{A}_{1}, \mathrm{~A}_{2} \ldots \mathrm{A}_{\mathrm{q}}\right\}$, and the propositions in $\Omega$ are finite, complete, and mutually exclusive, that is, the proposition $A$ can only take values in $\Omega$ at any time, and only one element in $\Omega$ can be taken as the value, then $\Omega$ is called recognition framework.

Next, the concept of the probability distribution function is given.

Definition 2 ([1]). Let $m$ be the probability distribution function on $2^{\Omega}$, then $m$ is defined as

$$
\left\{\begin{array}{l}
m(\Phi)=0 \\
\sum_{A \subseteq \Omega} m(A)=1 \\
0 \leq m(A) \leq 1
\end{array}\right.
$$

where $\phi$ represents the empty set, and $m(A)$ represents the support of evidence for the proposition $A$.

Based on the above definitions, D-S combination rule are given:

Definition 3 ([1]). According to the D-S evidence theory, the compound formula of $n$ pieces of evidence $E_{1}(A)$, $E_{2}(A), \ldots, E_{n}(A)$ is

$$
m(A)=\left\{\begin{array}{cc}
0 & \mathrm{~A}=\Phi \\
\frac{1}{1-k} \sum_{A_{s} \cap A_{u} \cdots \cap A_{v}=A} m_{1}\left(A_{s}\right) \cdot m_{2}\left(A_{u}\right) \ldots . . m_{n}\left(A_{v}\right) & \mathrm{A} \neq \Phi
\end{array} .\right.
$$

where $k$ represents the degree of conflict of $n$ pieces of evidence and is defined as

$$
k=\sum_{A_{s} \cap A_{u} \cap \ldots \cap A_{v}=\Phi} m_{1}\left(A_{s}\right) m_{2}\left(A_{u}\right) \ldots m_{n}\left(A_{v}\right) .
$$

Definition 4. Zadeh Paradox [19]. If there are two or more pieces of highly conflicting evidence in the system, the existence of conflicting evidence will cause the D-S evidence theory to fail or the combination result to be contrary to intuitive feelings.

Case 1: Assuming that the recognition frame of system is $\Omega=\left\{A_{1}, A_{2}, A_{3}\right\}$, and there are two pieces of evidence in the system:

$$
\begin{aligned}
& E_{1}: m_{1}\left(A_{1}\right)=1, m_{1}\left(A_{2}\right)=0, m_{1}\left(A_{3}\right)=0 \\
& E_{2}: m_{2}\left(A_{1}\right)=0, m_{2}\left(A_{2}\right)=1, m_{2}\left(A_{3}\right)=0
\end{aligned} .
$$


According to formula (3), $k=1$ is calculated, then in formula (2), $1-k=0$ causes D-S evidence theory to be invalid. Even if $E_{3}: m_{3}\left(A_{1}\right)=1, m_{3}\left(A_{2}\right)=0, m_{3}\left(A_{3}\right)=0, E_{4}: m_{4}\left(A_{1}\right)=1, m_{4}\left(A_{2}\right)=0$, $m_{4}\left(A_{3}\right)=0, \ldots, E_{n}: m_{n}\left(A_{1}\right)=1, m_{n}\left(A_{2}\right)=0, m_{n}\left(A_{3}\right)=0$ and other evidence is added to the system that is the same as $E_{1}$, the combination result still cannot be obtained because of $E_{2}$.

Case 2: Assuming that the recognition frame of system is $\Omega=\left\{A_{1}, A_{2}, A_{3}\right\}$, and there are two pieces of evidence in the system:

$$
\begin{aligned}
& E_{1}: m_{1}\left(A_{1}\right)=0.99, m_{1}\left(A_{2}\right)=0.01, m_{1}\left(A_{3}\right)=0 \\
& E_{2}: m_{2}\left(A_{1}\right)=0, m_{2}\left(A_{2}\right)=0.01, m_{2}\left(A_{3}\right)=0.99
\end{aligned} .
$$

We calculate $k=0.9999$ by formula (3), and then calculate $m\left(A_{1}\right)=0, m\left(A_{2}\right)=1, m_{3}\left(A_{3}\right)=0$ by formula (2). This result is obviously counterintuitive.

The maximum likelihood function and the maximum trust function are the two decision rules which are most commonly used in D-S evidence theory. In order to eliminate the influence of subjectivity on decision-making, the method proposed in this paper cancels the threshold setting and selects the proposition with the highest support as the final decision-making result. This paper uses the decision rule proposed by Li [20]:

Definition 5 ([20]). Decision rule can be defined as

$$
\left\{\begin{array}{l}
m\left(A_{i}\right)=\max \left\{m\left(A_{u}\right), A_{u} \subseteq \Omega\right\} \\
m\left(A_{j}\right)=\max \left\{m\left(A_{u}\right), A_{u} \subseteq\left(\Omega \backslash A_{i}\right)\right\} \\
m\left(A_{i}\right)-m\left(A_{j}\right)>0
\end{array} .\right.
$$

\section{Existing Modified Methods}

Researchers have two ways to improve D-S theory. One route is to improve the original evidence, and the other route is to improve the combination rule. The representative methods of the two routes are introduced below.

\subsection{Methods of Improving the Original Evidence}

The basic idea of methods of improving the original evidence is to keep the rule of evidence combination unchanged, weight the average of the $n$ pieces of evidence to be combined before evidence combination to obtain a new evidence, and then use D-S evidence combination rule to combine $n-1$ times for the new evidence to obtain the final result. In the method proposed by Murphy [5], the weight of all evidence is equal, that is, the weight of each evidence is $1 / n$. There are two steps as follows in the method:

Step 1: Calculate the arithmetic mean of $n$ pieces of evidence $E_{1}(A), E_{2}(A), \ldots, E_{n}(A)$ to get new evidence $E^{0}(A)$ :

$$
E^{0}(A)=\frac{1}{n}\left(E_{1}(A)+E_{2}(A)+\ldots+E_{n}(A)\right) .
$$

Step 2: After the calculation of $E^{0}(A)$, adopt formula (2) to combine new evidence $E^{0}(A)$ $n-1$ times.

\subsection{Methods of Improving the Combination Rule}

Another way to improve the D-S evidence theory is to improve the combination rule, rather than changing the evidence to be combined. The rule of evidence combination is as follows, which is proposed by Li [4]:

$$
\left\{\begin{array}{l}
m(\Phi)=0 \\
m(A)=\sum_{A_{s} \cap A_{u} \cap \ldots \cap A_{v}=A} m_{1}\left(A_{s}\right) \cdot m_{2}\left(A_{u}\right) \ldots . m_{n}\left(A_{v}\right)+k_{w} \cdot h(A) \forall A \neq \Phi .
\end{array}\right.
$$


where $k_{w}$ and $h(A)$ represent the coefficient of evidence conflict and the average degree of support for the proposition $A$ by each evidence, respectively. $k_{w}$ and $h(A)$ are given as follows:

$$
\begin{gathered}
k_{w}=\sum_{A_{s} \cap A_{u} \cap \ldots \cap A_{v}=\phi} m_{1}\left(A_{s}\right) \cdot m_{2}\left(A_{u}\right) . . . m_{n}\left(A_{v}\right) . \\
h(A)=\frac{1}{n} \sum_{1 \leq i \leq n} m_{i}(A) .
\end{gathered}
$$

\section{Combination Method for Conflicting Evidence Based on Historical Data}

\subsection{Data Definition and Recall}

Before the weight of evidence calculated, two types of data stored in the system need to be defined and called.

The first type of data is the evidence formed by each sensor within a period of time before time $t_{k+1}$ and the latest evidence to be combined formed at time $t_{k+1}$.

For research needs, this paper adopts the vector $E_{i}(A)_{t_{k}}$ to represent the observation result of the $i$ th sensor at time $t_{k}$ :

$$
E_{i}(A)_{t_{k}}=\left(m_{i}\left(A_{1}\right)_{t_{k}}, m_{i}\left(A_{2}\right)_{t_{k^{\prime}}} \ldots, m_{i}\left(A_{q}\right)_{t_{k}}\right) .
$$

where $m_{i}\left(A_{j}\right)_{t_{k}}$ represents the degree of support for the proposition $A_{j}$ by evidence $E_{i}$ and satisfies Equation (1):

$$
\left\{\begin{array}{l}
\sum_{j=1}^{q} m_{i}\left(A_{j}\right)_{t_{k}}=1 \\
0 \leq m_{i}\left(A_{j}\right)_{t_{k}} \leq 1
\end{array} .\right.
$$

The evidence $E_{i}(A)_{t_{1}}, E_{i}(A)_{t_{2}}, \ldots, E_{i}(A)_{t_{k}}$ formed by the $i$ th sensor in a period of time before time $t_{k+1}$ is historical data, which can be retrieved directly by querying system records.

$E_{i}(A)_{t_{k+1}}$ represents the latest and to-be-combined evidence observed by sensor at time $t_{k+1}$ :

$$
E_{i}(A)_{t_{k+1}}=\left(m_{i}\left(A_{1}\right)_{t_{k+1}}, m_{i}\left(A_{2}\right)_{t_{k+1}}, \ldots, m_{i}\left(A_{q}\right)_{t_{k+1}}\right) .
$$

where $m_{i}\left(A_{j}\right)_{t_{k+1}}$ represents the degree of support for the proposition $A_{j}$ by evidence $E_{i}$ and satisfies Equation (1).

The latest evidence to be combined which is observed by $n$ sensors at time $t_{k+1}$ is received and stored by the system, and can be directly retrieved during subsequent combination.

The second type of data is the true result vector corresponding to each sensor when observing evidence for a period of time before time $t_{k+1}$. The $i$ th sensor forms evidence $E_{i}(A)_{t_{1}}, E_{i}(A)_{t_{2}}, \ldots, E_{i}(A)_{t_{k}}$ within a period before time $t_{k+1}$, and the corresponding true result vectors are $R_{i}(A)_{t_{1}}, R_{i}(A)_{t_{2}}, \ldots$, $R_{i}(A)_{t_{k}}$ respectively.

$R_{i}(A)_{t_{k}}$ represents the true result vector at time $t_{k}$ :

$$
R_{i}(A)_{t_{k}}=\left(r_{i}\left(A_{1}\right)_{t_{k}}, r_{i}\left(A_{2}\right)_{t_{k}}, \ldots, r_{i}\left(A_{q}\right)_{t_{k}}\right) .
$$

where $r_{i}\left(A_{j}\right)_{t_{k}}$ not only satisfies Equation (1), but also can only be 0 or 1 .

Therefore, unlike the vector $E_{i}(A)_{t_{k^{\prime}}}$, all components of the vector $R_{i}(A)_{t_{k}}$ can only be 0 or 1 , and only one component of the vector $R_{i}(A)_{t_{k}}$ can be 1 . The true result vector can be recorded by the system after the decision and updated within a period of time after each decision is completed. The true result vectors can be retrieved from the system. The quantity of true result vectors retrieved is represented by $l_{i}$, and $l_{i} \geq 0$. When $l_{i}=0$, it means that the sensor newly enters the system or the method proposed in this paper is not used in the system for evidence combination. 
$R_{i}(A)_{t_{k+1}}$ represents the true result vector at time $t_{k+1}$ :

$$
R_{i}(A)_{t_{k+1}}=\left(r_{i}\left(A_{1}\right)_{t_{k+1}}, r_{i}\left(A_{2}\right)_{t_{k+1}}, \ldots, r_{i}\left(A_{q}\right)_{t_{k+1}}\right) .
$$

where $r_{i}\left(A_{j}\right)_{t_{k+1}}$ not only satisfies Equation (1), but also can only be 0 or 1 and because the true result vector at that moment is unique

$$
R_{1}(A)_{t_{k+1}}=R_{2}(A)_{t_{k+1}}=\ldots=R_{n}(A)_{t_{k+1}} .
$$

However, the true result vector $R_{i}(A)_{t_{k+1}}$ is unknown before decisions have been made. Decision makers need to find the proposition $A$ with the highest probability value.

\subsection{Calculation of Weights of Evidence}

In the method proposed in this paper, each evidence to be combined is weighted. The weight of the evidence $E_{i}(A)_{t_{k+1}}$ is $\omega_{i}$. Employing the spectral angle cosine function [21], the weight of the evidence $E_{i}(A)_{t_{k+1}}$ is

$$
\omega_{i}=\left\{\begin{array}{c}
1 / n \quad l_{i}=0 \\
\frac{\sum_{t_{k}=1}^{l_{i}} \cos \left(M_{i}(A)_{t_{k^{\prime}}} R_{i}(A)_{t_{k}}\right)}{l_{i}}=\frac{1}{l_{i}}\left(\sum_{t_{k}=1}^{l_{i}} \frac{\sum_{j=1}^{q} m_{i}\left(A_{j}\right)_{t_{k}} r_{i}\left(A_{j}\right)_{t_{k}}}{\sqrt{\sum_{j=1}^{q} m_{i}{ }^{2}\left(A_{j}\right)_{t_{k}}} \sqrt{\sum_{j=1}^{q} r_{i}^{2}\left(A_{j}\right)_{t_{k}}}}\right) l_{i} \geq 1 .
\end{array}\right.
$$

where the value of $\omega_{i}$ is set as the average value when $l_{i}=0$, to ensure that the system can perform evidence combination without the true result vector $R_{i}(A)_{t_{k}}$.

Then, the credibility degree of evidence $E_{i}(A)_{t_{k+1}}$ can be defined by $\omega_{i}$ :

$$
\overline{\omega_{i}}=\left\{\begin{array}{cc}
1 / n & \sum_{i=1}^{n} \omega_{i}=0 \\
\frac{\omega_{i}}{\sum_{i=1}^{n} \omega_{i}} & \sum_{i=1}^{n} \omega_{i} \neq 0
\end{array} .\right.
$$

\subsection{Generation of New Evidence}

After obtaining the weight $\overline{\omega_{i}}$ corresponding to each evidence $E_{i}(A)_{t_{k+1}}$ to be combined, we can obtain new evidence $E^{0}(A)$ by weighted average method.

$$
E^{0}(A)=\overline{\omega_{1}} E_{1}(A)_{t_{k+1}}+\overline{\omega_{2}} E_{2}(A)_{t_{k+1}}+\ldots+\overline{\omega_{n}} E_{n}(A)_{t_{k+1}} .
$$

$E^{0}(A)$ replaces the original evidence $E_{1}(A)_{t_{k+1}}, E_{2}(A)_{t_{k+1}}, . . E_{n}(A)_{t_{k+1}}$, and is used in subsequent evidence combination.

\subsection{Combination of New Evidence}

This paper adopts formula (2) to combine new evidence $E^{0}(A)$. Because there are $n$ pieces of original evidence in the system, the number of times to combine is $n-1$. In the first combination, $E^{0}(A)$ and $E^{0}(A)$ are combined using formula (2) to obtain new evidence $E^{1}(A)$. In the second combination, formula (2) is used to combine evidence $E^{0}(A)$ and $E^{1}(A)$ obtained in the first combination to obtain another new evidence $E^{2}(A)$, and so on. At the $(n-1)$ th time, that is, in the last combination, formula (2) is still used to combine evidence $E^{0}(A)$ and $E^{n-1}(A)$ obtained in the (n-2)th combination. Combination is performed $n-1$ times by formula (2), and finally $E^{n}(A)$ is obtained. 


\subsection{Decision Rule}

If $A_{i}$ satisfies formula (6) in $E^{n}(A)$, it is the final decision result. If there is no $A_{i}$ that satisfies the condition of formula (6) and there is a unique $i_{0}$ that satisfies formula (20), then the decision can be made according to formula (21) and $A_{i}$ satisfying formula (21) is the final decision result:

$$
\begin{gathered}
\left\{\begin{array}{l}
\omega_{\mathrm{i}_{0}}=\max _{1 \leq i \leq n}\left\{\omega_{\mathrm{i}}\right\} \\
1 \leq i_{0} \leq n
\end{array} .\right. \\
\left\{\begin{array}{l}
m\left(A_{i}\right)=\max _{1 \leq u \leq q}\left\{m_{i_{0}}\left(A_{u}\right)_{t_{k+1}}\right\} \\
m\left(A_{j}\right)=\max _{1 \leq u \leq q}\left\{m_{i_{0}}\left(A_{u}\right)_{t_{k+1}}, u \neq i\right\} . \\
m\left(A_{i}\right)-m\left(A_{j}\right)>0
\end{array}\right.
\end{gathered}
$$

\subsection{Update of Real Result}

After making a decision, the system updates the data according to the actual situation. If real result is available, the system updates the value of $R_{i}(A)_{t_{k+1}}$. Moreover, $E_{1}(A)_{t_{k+1}}, E_{2}(A)_{t_{k+1}}, \ldots E_{n}(A)_{t_{k+1}}$ and $R_{i}(A)_{t_{k+1}}$ become new historical data available. Otherwise, the system sets the value of $R_{i}(A)_{t_{k+1}}$ to empty. The updated result is stored in the system for subsequent query and recall.

\subsection{Flow of the Proposed Method}

In general, the specific implementation of the proposed method can be summarized:

Step 1: Define data and retrieve historical data stored in the system. These data include not only the new evidence to be combined, but also the formed evidence and the corresponding true result vector within a period before $t_{k+1}$. Combining evidence based on historical data is a major innovation of this paper.

Step 2: Adopt spectral angle cosine function to calculate the credibility (i.e., weight) of each piece of evidence to be combined based on historical data called in the first step and then normalize it, by which another innovation point of this paper is marked.

Step 3: Weight average all the pieces of evidence to be combined and get new evidence $E^{0}(A)$.

Step 4: Adopt D-S combination rule to combine $E^{0}(A) n-1$ times to get $E^{n}(A)$.

Step 5: Select $A_{i}$ with maximum credibility according to decision rule. When there are two or more propositions with the maximum support in the combination results, decision makers can select the proposition with the highest support as the final decision result in the evidence with the largest weight based on historical data. Modified decision rule is another innovation point of this paper.

Step 6: Update and store $R_{i}(A)_{t_{k+1}}$ according to the actual result available to form new historical data that can be accessed and called.

\section{Simulation Result and Analysis}

\subsection{Basic Data}

To verify the effectiveness of the method in this paper, two simulation scenarios are selected. One scenario is where the good evidence is in the majority, and the other scenario is where the bad evidence is in the majority. In the first scenario, the accuracy of the evidence observed by most sensors is high, and the error of the evidence observed by a few sensors is large, which means that evidence $E_{2}$ is the outlier. In the second scenario, the accuracy of the evidence observed by a few sensors is high, and the error of the evidence observed by most sensors is large, which means that evidence $E_{1}, E_{3}, E_{4}$ and $E_{5}$ are the outliers. Each scenario is divided into two stages. The time of evidence collection in the first stage is set to $t_{k+1}$, and the time of evidence collection in the second stage is set to $t_{k+2}$. In order to facilitate the comparative analysis with other existing methods, the evidence used at $t_{k+1}$ and time $t_{k+2}$ 
are derived from numerical examples adopted by Deng [6]. That is, there are five sensors in the system to observe the three targets $A_{1}, A_{2}$ and $A_{3}$.

$$
\begin{aligned}
& E_{1}(A)_{t_{k+1}}=\left(m_{1}\left(A_{1}\right)_{t_{k+1}}, m_{1}\left(A_{2}\right)_{t_{k+1}}, m_{1}\left(A_{3}\right)_{t_{k+1}}\right)=(0.50,0.20,0.30) . \\
& E_{2}(A)_{t_{k+1}}=\left(m_{2}\left(A_{1}\right)_{t_{k+1}}, m_{2}\left(A_{2}\right)_{t_{k+1}}, m_{2}\left(A_{3}\right)_{t_{k+1}}\right)=(0.00,0.90,0.10) . \\
& E_{3}(A)_{t_{k+1}}=\left(m_{3}\left(A_{1}\right)_{t_{k+1}}, m_{3}\left(A_{2}\right)_{t_{k+1}}, m_{3}\left(A_{3}\right)_{t_{k+1}}\right)=(0.55,0.10,0.35) . \\
& E_{4}(A)_{t_{k+1}}=\left(m_{4}\left(A_{1}\right)_{t_{k+1}}, m_{4}\left(A_{2}\right)_{t_{k+1}}, m_{4}\left(A_{3}\right)_{t_{k+1}}\right)=(0.55,0.10,0.35) . \\
& E_{5}(A)_{t_{k+1}}=\left(m_{5}\left(A_{1}\right)_{t_{k+1}}, m_{5}\left(A_{2}\right)_{t_{k+1}}, m_{5}\left(A_{3}\right)_{t_{k+1}}\right)=(0.55,0.10,0.35) . \\
& E_{1}(A)_{t_{k+2}}=\left(m_{1}\left(A_{1}\right)_{t_{k+2}}, m_{1}\left(A_{2}\right)_{t_{k+2}}, m_{1}\left(A_{3}\right)_{t_{k+2}}\right)=(0.50,0.20,0.30) . \\
& E_{2}(A)_{t_{k+2}}=\left(m_{2}\left(A_{1}\right)_{t_{k+2}}, m_{2}\left(A_{2}\right)_{t_{k+2}}, m_{2}\left(A_{3}\right)_{t_{k+2}}\right)=(0.00,0.90,0.10) . \\
& E_{3}(A)_{t_{k+2}}=\left(m_{3}\left(A_{1}\right)_{t_{k+2}}, m_{3}\left(A_{2}\right)_{t_{k+2}}, m_{3}\left(A_{3}\right)_{t_{k+2}}\right)=(0.55,0.10,0.35) . \\
& E_{4}(A)_{t_{k+2}}=\left(m_{4}\left(A_{1}\right)_{t_{k+2}}, m_{4}\left(A_{2}\right)_{t_{k+2}}, m_{4}\left(A_{3}\right)_{t_{k+2}}\right)=(0.55,0.10,0.35) . \\
& E_{5}(A)_{t_{k+2}}=\left(m_{5}\left(A_{1}\right)_{t_{k+2}}, m_{5}\left(A_{2}\right)_{t_{k+2}}, m_{5}\left(A_{3}\right)_{t_{k+2}}\right)=(0.55,0.10,0.35) .
\end{aligned}
$$

It is assumed that the system adopts the proposed method from $t_{k+1}$, and the real result of $t_{k+1}$ can be known after decision. The effectiveness and priority of the proposed method is verified by comparing it with D-S [1], Yager [2], Sun [3], Li [4], Murphy [5] and Deng [6].

\subsection{Analysis of Result in Cases Where Good Evidence Predominate}

\subsubsection{First Stage Result}

At the first stage in cases where good evidence is dominant, there is no historical data in the system. Therefore, the weight of each evidence obtained according to formula (17) should be equal, and normalized by formula (18). When there are only two pieces of evidence $E_{1}(A)_{t_{k+1}}$ and $E_{2}(A)_{t_{k+1}}$ in the system, $\overline{\omega_{1}}=\overline{\omega_{2}}=1 / 2$. When there are three pieces of evidence $E_{1}(A)_{t_{k+1}}, E_{2}(A)_{t_{k+1}}$ and $E_{3}(A)_{t_{k+1}}$ in the system, $\overline{\omega_{1}}=\overline{\omega_{2}}=\overline{\omega_{3}}=1 / 3$. When there are four pieces of evidence $E_{1}(A)_{t_{k+1}}, E_{2}(A)_{t_{k+1}}, E_{3}(A)_{t_{k+1}}$ and $E_{4}(A)_{t_{k+1}}$ in the system, $\overline{\omega_{1}}=\overline{\omega_{2}}=\overline{\omega_{3}}=\overline{\omega_{4}}=1 / 4$. When there are five pieces of evidence $E_{1}(A)_{t_{k+1}}, E_{2}(A)_{t_{k+1}}, E_{3}(A)_{t_{k+1}}, E_{4}(A)_{t_{k+1}}$ and $E_{5}(A)_{t_{k+1}}$ in the system, $\overline{\omega_{1}}=\overline{\omega_{2}}=\overline{\omega_{3}}=\overline{\omega_{4}}=\overline{\omega_{5}}=1 / 5$. It is clear that the true target observed by all sensors is $A 1$ with good evidence in the majority. The simulation results are shown in Table 1.

The following four conclusions can be drawn from Table 1:

Conclusion 1: From the simulation result, in the case of two pieces of evidence $E_{1}, E_{2}$ and three pieces of evidence $E_{1}, E_{2}$, and $E_{3}$, the combination result of D-S [1] reject $A 1$ and support $A 2$, which is inconsistent with intuition. With the successive addition of evidence $E_{4}$ and $E_{5}, A 1$ is still rejected, and the combination result changes from supporting $A 2$ to supporting $A 3$. Obviously, this is the problem of "Zadeh Paradox" mentioned before [20] and can be solved effectively by the proposed method.

Conclusion 2: In this case, "not being contrary to intuitive feeling" and "not being contrary to the facts" are the same. When there are only two pieces of evidence $E_{1}$ and $E_{2}$, all the methods fail to correctly identify the true target. When evidence $E_{3}$ is added, Deng [6] first identify the true target. When adding evidence $E_{4}$, all methods except D-S [1], Yager [2] and Sun [3] identify the true target. When evidence $E_{5}$ is added at the end, D-S [1], Yager [2] and Sun [3] are still unable to identify the true target. However, the remaining methods not only identify the real target, but also increase the support for the real target $A 1$. This is because when good evidence is gradually increasing, the ability of the system to resist interference is getting stronger and stronger, making the real target easier to identify. 
Conclusion 3: The calculation results of the proposed method are consistent with Murphy [5] in this case. This is because in the absence of historical data, the proposed method cannot weight evidence based on the angle cosine, but only simple arithmetic average weighting.

Conclusion 4: The method proposed in this paper can make effective decisions based on the combination of evidence. When there is only one proposition with the maximum support, this proposition is selected as the final decision result. When there are two or more propositions with the maximum support, the proposition with the highest support is selected as the final decision result in the evidence with the largest weight.

Table 1. Simulation result of fist stage in cases where good evidence predominates.

\begin{tabular}{|c|c|c|c|c|c|c|c|}
\hline Evidence & Methods & $m(A 1)$ & $m(A 2)$ & $m(A 3)$ & $m(X)$ & Predicted Target & True Target \\
\hline \multirow{7}{*}{$E 1, E 2$} & D-S [1] & 0 & 0.8571 & 0.1429 & & $A 2$ & $A 1$ \\
\hline & Yager [2] & 0 & 0.18 & 0.03 & 0.79 & $X$ & $A 1$ \\
\hline & Sun [3] & 0.090 & 0.377 & 0.102 & 0.431 & $A 2$ & $A 1$ \\
\hline & $\operatorname{Li}[4]$ & 0.1975 & 0.6145 & 0.1880 & & $A 2$ & $A 1$ \\
\hline & Murphy [5] & 0.1543 & 0.7469 & 0.0988 & & $A 2$ & $A 1$ \\
\hline & Deng [6] & 0.1543 & 0.7469 & 0.0988 & & $A 2$ & $A 1$ \\
\hline & Proposed & 0.1543 & 0.7469 & 0.0988 & & $A 2$ & $A 1$ \\
\hline \multirow{7}{*}{$E 1, E 2, E 3$} & D-S [1] & 0 & 0.6316 & 0.3684 & & $A 2$ & $A 1$ \\
\hline & Yager [2] & 0 & 0.018 & 0.0105 & 0.9715 & $X$ & $A 1$ \\
\hline & Sun [3] & 0.175 & 0.218 & 0.135 & 0.472 & $X$ & $A 1$ \\
\hline & $\operatorname{Li}[4]$ & 0.3400 & 0.4066 & 0.2534 & & $A 2$ & $A 1$ \\
\hline & Murphy [5] & 0.3500 & 0.5224 & 0.1276 & & $A 2$ & $A 1$ \\
\hline & Deng [6] & 0.5816 & 0.2439 & 0.1745 & & $A 1$ & $A 1$ \\
\hline & Proposed & 0.3500 & 0.5224 & 0.1276 & & $A 2$ & $A 1$ \\
\hline \multirow{7}{*}{$E 1, E 2, E 3, E 4$} & D-S [1] & 0 & 0.3288 & 0.6712 & & $A 3$ & $A 1$ \\
\hline & Yager [2] & 0 & 0.0018 & 0.00368 & 0.99452 & $X$ & $A 1$ \\
\hline & Sun [3] & 0.194 & 0.160 & 0.137 & 0.509 & $X$ & $A 1$ \\
\hline & $\mathrm{Li}[4]$ & 0.3978 & 0.3250 & 0.2772 & & $A 1$ & $A 1$ \\
\hline & Murphy [5] & 0.6027 & 0.2627 & 0.1346 & & $A 1$ & $A 1$ \\
\hline & Deng [6] & 0.8060 & 00482 & 0.1458 & & $A 1$ & $A 1$ \\
\hline & Proposed & 0.6027 & 0.2627 & 0.1346 & & A1 & $A 1$ \\
\hline \multirow{7}{*}{$E 1, E 2, E 3, E 4, E 5$} & D-S [1] & 0 & 0.1288 & 0.8772 & & $A 3$ & $A 1$ \\
\hline & Yager [2] & 0 & 0.00018 & 0.00129 & 0.99853 & $X$ & $A 1$ \\
\hline & Sun [3] & 0.211 & 0.138 & 0.144 & 0.507 & $X$ & $A 1$ \\
\hline & $\operatorname{Li}[4]$ & 0.4294 & 0.2798 & 0.2909 & & $A 1$ & $A 1$ \\
\hline & Murphy [5] & 0.7958 & 0.0932 & 0.1110 & & $A 1$ & $A 1$ \\
\hline & Deng [6] & 0.8909 & 0.0086 & 0.1005 & & $A 1$ & $A 1$ \\
\hline & Proposed & 0.7958 & 0.0932 & 0.1110 & & $A 1$ & $A 1$ \\
\hline
\end{tabular}

\subsubsection{Second Stage Result}

At the second stage in cases where good evidence is dominant, there is historical data in the system including $E_{1}(A)_{t_{k+1}}, E_{2}(A)_{t_{k+1}}, E_{3}(A)_{t_{k+1}}, E_{4}(A)_{t_{k+1}}, E_{5}(A)_{t_{k+1}}, R_{i}(A)_{t_{k+1}}$, etc. Since the real target observed by all sensors is $A 1$, the true result vector $R_{i}(A)_{t_{k+1}}=(1,0,0)$ is calculated according to formula (15) based on the historical data produced in the first stage. Therefore, $\omega_{1}=0.8111, \omega_{2}=0$, $\omega_{3}=\omega_{4}=\omega_{5}=0.8339$ can be obtained according to formula (17) and normalized by formula (18). When there are only evidence $E_{1}(A)_{t_{k+2}}$ and $E_{2}(A)_{t_{k+2}}$ in the system, $\overline{\omega_{1}}=1, \overline{\omega_{2}}=0$. When there are evidence $E_{1}(A)_{t_{k+2}}, E_{2}(A)_{t_{k+2}}$ and $E_{3}(A)_{t_{k+2}}$ in the system, $\overline{\omega_{1}}=0.4931, \overline{\omega_{2}}=0, \overline{\omega_{3}}=0.5069$. When there are evidence $E_{1}(A)_{t_{k+2}}, E_{2}(A)_{t_{k+2}}, E_{3}(A)_{t_{k+2}}$ and $E_{4}(A)_{t_{k+2}}$ in the system, $\overline{\omega_{1}}=0.3272, \overline{\omega_{2}}=0$, $\overline{\omega_{3}}=\overline{\omega_{4}}=0.3364$. When there are evidence $E_{1}(A)_{t_{k+2}}, E_{2}(A)_{t_{k+2}}, E_{3}(A)_{t_{k+2}}, E_{4}(A)_{t_{k+2}}$ and $E_{5}(A)_{t_{k+2}}$ in the system, $\overline{\omega_{1}}=0.2448, \overline{\omega_{2}}=0, \overline{\omega_{3}}=\overline{\omega_{4}}=\overline{\omega_{5}}=0.2517$. It is clear that the true target observed by all sensors is $A 1$ with good evidence in the majority. The simulation result is shown in Table 2. 
Table 2. Simulation result of second stage in cases where good evidence predominates.

\begin{tabular}{|c|c|c|c|c|c|c|c|}
\hline Evidence & Methods & $m(A 1)$ & $m(A 2)$ & $m(A 3)$ & $m(X)$ & Predicted Target & True Target \\
\hline \multirow{7}{*}{$E 1, E 2$} & D-S [1] & 0 & 0.8571 & 0.1429 & & $A 2$ & $A 1$ \\
\hline & Yager [2] & 0 & 0.18 & 0.03 & 0.79 & $X$ & $A 1$ \\
\hline & Sun [3] & 0.090 & 0.377 & 0.102 & 0.431 & $A 2$ & $A 1$ \\
\hline & $\operatorname{Li}[4]$ & 0.1975 & 0.6145 & 0.1880 & & $A 2$ & $A 1$ \\
\hline & Murphy [5] & 0.1543 & 0.7469 & 0.0988 & & $A 2$ & $A 1$ \\
\hline & Deng [6] & 0.1543 & 0.7469 & 0.0988 & & $A 2$ & $A 1$ \\
\hline & Proposed & 0.6579 & 0.1053 & 0.2368 & & $A 1$ & $A 1$ \\
\hline \multirow{7}{*}{$E 1, E 2, E 3$} & D-S [1] & 0 & 0.6316 & 0.3684 & & $A 2$ & $A 1$ \\
\hline & Yager [2] & 0 & 0.018 & 0.0105 & 0.9715 & $X$ & $A 1$ \\
\hline & Sun [3] & 0.175 & 0.218 & 0.135 & 0.472 & $X$ & $A 1$ \\
\hline & $\operatorname{Li}[4]$ & 0.3400 & 0.4066 & 0.2534 & & $A 2$ & $A 1$ \\
\hline & Murphy [5] & 0.3500 & 0.5224 & 0.1276 & & $A 2$ & $A 1$ \\
\hline & Deng [6] & 0.5816 & 0.2439 & 0.1745 & & $A 1$ & $A 1$ \\
\hline & Proposed & 0.7934 & 0.0182 & 0.1884 & & $A 1$ & $A 1$ \\
\hline \multirow{7}{*}{$E 1, E 2, E 3, E 4$} & D-S [1] & 0 & 0.3288 & 0.6712 & & $A 3$ & $A 1$ \\
\hline & Yager [2] & 0 & 0.0018 & 0.00368 & 0.99452 & $X$ & $A 1$ \\
\hline & Sun [3] & 0.194 & 0.160 & 0.137 & 0.509 & $X$ & $A 1$ \\
\hline & $\operatorname{Li}[4]$ & 0.3978 & 0.3250 & 0.2772 & & $A 1$ & $A 1$ \\
\hline & Murphy [5] & 0.6027 & 0.2627 & 0.1346 & & $A 1$ & $A 1$ \\
\hline & Deng [6] & 0.8060 & 00482 & 0.1458 & & $A 1$ & $A 1$ \\
\hline & Proposed & 0.8646 & 0.0033 & 0.1321 & & $A 1$ & $A 1$ \\
\hline \multirow{7}{*}{$E 1, E 2, E 3, E 4, E 5$} & D-S [1] & 0 & 0.1288 & 0.8772 & & $A 3$ & $A 1$ \\
\hline & Yager [2] & 0 & 0.00018 & 0.00129 & 0.99853 & $X$ & $A 1$ \\
\hline & Sun [3] & 0.211 & 0.138 & 0.144 & 0.507 & $X$ & $A 1$ \\
\hline & $\operatorname{Li}[4]$ & 0.4294 & 0.2798 & 0.2909 & & $A 1$ & $A 1$ \\
\hline & Murphy [5] & 0.7958 & 0.0932 & 0.1110 & & $A 1$ & $A 1$ \\
\hline & Deng [6] & 0.8909 & 0.0086 & 0.1005 & & $A 1$ & $A 1$ \\
\hline & Proposed & 0.9104 & 0.0006 & 0.0890 & & $A 1$ & $A 1$ \\
\hline
\end{tabular}

In addition to the method in this paper being able to effectively solve the "Zadeh Paradox" problem [20] and help decision makers make effective decisions, two other analyses following can be got from Table 2:

Conclusion 5: "Not being contrary to intuitive feeling" is consistent with "not being contrary to the facts". From the simulation result, when there are only evidence $E_{1}$ and $E_{2}$, all the methods fail to correctly identify the true target except the proposed method. When evidence $E_{3}$ is added, only Deng [6] and the proposed method can identify the true target. When adding evidence $E_{4}$, all methods except D-S [1], Yager [2] and Sun [3] can identify the true target. When evidence $E_{5}$ is added at the end, D-S [1], Yager [2] and Sun [3] are still unable to identify the true target. However, the remaining methods not only identify the real target, but also increase the support for the real target $A 1$. This is because when good evidence is gradually increasing, the ability of the system to resist interference is getting stronger and stronger, making real target easier to identify.

Conclusion 6: The method proposed in this paper can achieve convergence when there are two pieces of evidence in the case where there is historical data and good evidence is the majority, as well as has the fastest convergence speed. The reason is that the method proposed in this paper can strengthen or weaken the evidence to be combined in the second stage based on the historical data collected in the first stage.

\subsection{Analysis of Result in Cases Where Bad Evidence Predominates}

\subsubsection{First Stage Result}

At the first stage in cases where bad evidence is dominant, there is also no historical data in the system. In this case, the weight of each piece of evidence is the same as in the first stage of the case where good evidence is the majority. It is clear that the true target observed by all sensors is $A 2$ with bad evidence in the majority. The simulation result is shown in Table 3. 
Table 3. Simulation result of fist stage in cases where bad evidence predominates.

\begin{tabular}{|c|c|c|c|c|c|c|c|}
\hline Evidence & Methods & $m(A 1)$ & $m(A 2)$ & $m(A 3)$ & $m(X)$ & Predicted Target & True Target \\
\hline \multirow{7}{*}{$E 1, E 2$} & D-S [1] & 0 & 0.8571 & 0.1429 & & $A 2$ & $A 2$ \\
\hline & Yager [2] & 0 & 0.18 & 0.03 & 0.79 & $X$ & $A 2$ \\
\hline & Sun [3] & 0.090 & 0.377 & 0.102 & 0.431 & $A 2$ & $A 2$ \\
\hline & $\mathrm{Li}[4]$ & 0.1975 & 0.6145 & 0.1880 & & $A 2$ & $A 2$ \\
\hline & Murphy [5] & 0.1543 & 0.7469 & 0.0988 & & $A 2$ & $A 2$ \\
\hline & Deng [6] & 0.1543 & 0.7469 & 0.0988 & & $A 2$ & $A 2$ \\
\hline & Proposed & 0.1543 & 0.7469 & 0.0988 & & $A 2$ & $A 2$ \\
\hline \multirow{7}{*}{$E 1, E 2, E 3$} & D-S [1] & 0 & 0.6316 & 0.3684 & & $A 2$ & $A 2$ \\
\hline & Yager [2] & 0 & 0.018 & 0.0105 & 0.9715 & $X$ & $A 2$ \\
\hline & Sun [3] & 0.175 & 0.218 & 0.135 & 0.472 & $X$ & $A 2$ \\
\hline & $\operatorname{Li}[4]$ & 0.3400 & 0.4066 & 0.2534 & & $A 2$ & $A 2$ \\
\hline & Murphy [5] & 0.3500 & 0.5224 & 0.1276 & & $A 2$ & $A 2$ \\
\hline & Deng [6] & 0.5816 & 0.2439 & 0.1745 & & $A 1$ & $A 2$ \\
\hline & Proposed & 0.3500 & 0.5224 & 0.1276 & & $A 2$ & $A 2$ \\
\hline \multirow{7}{*}{$E 1, E 2, E 3, E 4$} & D-S [1] & 0 & 0.3288 & 0.6712 & & $A 3$ & $A 2$ \\
\hline & Yager [2] & 0 & 0.0018 & 0.00368 & 0.99452 & $X$ & $A 2$ \\
\hline & Sun [3] & 0.194 & 0.160 & 0.137 & 0.509 & $X$ & $A 2$ \\
\hline & $\operatorname{Li}[4]$ & 0.3978 & 0.3250 & 0.2772 & & $A 1$ & $A 2$ \\
\hline & Murphy [5] & 0.6027 & 0.2627 & 0.1346 & & $A 1$ & $A 2$ \\
\hline & Deng [6] & 0.8060 & 00482 & 0.1458 & & $A 1$ & $A 2$ \\
\hline & Proposed & 0.6027 & 0.2627 & 0.1346 & & $A 1$ & $A 2$ \\
\hline \multirow{7}{*}{$E 1, E 2, E 3, E 4, E 5$} & D-S [1] & 0 & 0.1288 & 0.8772 & & $A 3$ & $A 2$ \\
\hline & Yager [2] & 0 & 0.00018 & 0.00129 & 0.99853 & $X$ & $A 2$ \\
\hline & Sun [3] & 0.211 & 0.138 & 0.144 & 0.507 & $X$ & $A 2$ \\
\hline & $\operatorname{Li}[4]$ & 0.4294 & 0.2798 & 0.2909 & & $A 1$ & $A 2$ \\
\hline & Murphy [5] & 0.7958 & 0.0932 & 0.1110 & & $A 1$ & $A 2$ \\
\hline & Deng [6] & 0.8909 & 0.0086 & 0.1005 & & $A 1$ & $A 2$ \\
\hline & Proposed & 0.7958 & 0.0932 & 0.1110 & & $A 1$ & $A 2$ \\
\hline
\end{tabular}

In addition to the method in this paper being able to effectively solve the "Zadeh Paradox" problem [20] and help decision makers make effective decisions, four other conclusions following can be drawn from Table 3:

Conclusion 7: "Not being contrary to intuitive feeling" is not consistent with "not being contrary to the facts". Although the combination results more and more satisfy the principle of "not being contrary to intuitive feeling", but increasingly do not satisfy the principle of "not being contrary to the facts", the deviation from the true target $A 2$ is larger and larger. From the simulation result, when there are only evidence $E_{1}$ and $E_{2}$, all the methods except Yager [2] can correctly identify the real target. When there are evidence $E_{1}, E_{2}$, and $E_{3}$, Yager [2], Sun [3] and Deng [6] have incorrect recognition. DS [1], Li [4], Murphy [5] and the proposed method still correctly recognize true target. However, DS [1], Li [4], Murphy [5] and the proposed method still correctly identify the real target. When there are four pieces of evidence $E_{1}, E_{2}, E_{3}, E_{4}$ and five pieces of evidence $E_{1}, E_{2}, E_{3}, E_{4}, E_{5}$ in the system, all the methods fail to identify the real target. In this case, the effectiveness of the proposed method is the same as that of D-S [1], Li [4] and Murphy [5] and better than that of Yager [2], Sun [3] and Deng [6]

Conclusion 8: All the methods suffer from performance degradation with the increasement of bad evidence. This is because the bad evidence is gradually increasing, the system is getting more and more interference, and the effectiveness of various methods is getting worse and worse. It presents the "minority obeying majority" problem that is common in all current conflict evidence combination methods. The existence of the "minority obedience to majority" problem weakens the role of good evidence and magnifies the influence of bad evidence, which is why this article proposes to distinguish between different sources of evidence. Evidence collected by sensors with good performance and low environmental interference is obviously more reliable. Evidence collected by sensors with poor performance and high environmental interference often has low credibility. When combining evidence, it is of strong practical significance to be able to distinguish and assign weights to the evidence from different sources. 
Conclusion 9: It is not difficult to see that, with the increase of bad evidence, compared with Sun [3], Li [4], Murphy [5] and the method in this paper, the degree of support for true target of Deng [6] decreases faster. This is because Sun [3], Li [4], Murphy [5] and the proposed method are based on the arithmetic mean method, while Deng [6] weights the evidence based on the similarity between the evidence to be combined. When bad evidence is increasing, the performance of these methods decreases rapidly due to the rapid increase of errors. Therefore, when weighting the evidence, the proposed method is based on the accuracy of historical observation data rather than the similarity between the evidence to be combined.

Conclusion 10: The calculation result of the proposed method is consistent with Murphy [5] in this case. This is because in the absence of historical data, the proposed method cannot weight evidence based on the angle cosine, but only simple arithmetic average weighting.

\subsubsection{Second Stage Result}

At the second stage in cases where bad evidence is dominant, there is historical data in the system including $E_{1}(A)_{t_{k+1}}, E_{2}(A)_{t_{k+1}}, E_{3}(A)_{t_{k+1}}, E_{4}(A)_{t_{k+1}}, E_{5}(A)_{t_{k+1}}, R_{i}(A)_{t_{k+1}}$, etc. Since the real target observed by all sensors is $A 2$, the true result vector $R_{i}(A)_{t_{k+1}}=(0,1,0)$ is calculated according to formula (15). Therefore, $\omega_{1}=0.3244, \omega_{2}=0.9939, \omega_{3}=\omega_{4}=\omega_{5}=0.1516$ can be obtained according to formula (17) and normalized by formula (18). When there are only two pieces of evidence $E_{1}(A)_{t_{k+2}}$ and $E_{2}(A)_{t_{k+2}}$ in the system, $\overline{\omega_{1}}=0.2461, \overline{\omega_{2}}=0.7539$. When there are evidence $E_{1}(A)_{t_{k+2}}, E_{2}(A)_{t_{k+2}}$ and $E_{3}(A)_{t_{k+2}}$ in the system, $\overline{\omega_{1}}=0.2207, \overline{\omega_{2}}=0.6761, \overline{\omega_{3}}=0.1031$. When there are evidence in the system, $\overline{\omega_{1}}=0.2001, \overline{\omega_{2}}=0.6129, \overline{\omega_{3}}=\overline{\omega_{4}}=0.0935$. When there are five pieces of evidence in the system, $\overline{\omega_{1}}=0.1830, \overline{\omega_{2}}=0.5605, \overline{\omega_{3}}=\overline{\omega_{4}}=\overline{\omega_{5}}=0.0855$. It is clear that the true target observed by all sensors is $A 2$ with bad evidence in the majority. The simulation result is shown in Table 4.

Table 4. Simulation result of second stage in cases where bad evidence predominates.

\begin{tabular}{|c|c|c|c|c|c|c|c|}
\hline Evidence & Methods & $m(A 1)$ & $m(A 2)$ & $m(A 3)$ & $m(X)$ & Predicted Target & True Target \\
\hline \multirow{7}{*}{$E 1, E 2$} & D-S [1] & 0 & 0.8571 & 0.1429 & & $A 2$ & $A 2$ \\
\hline & Yager [2] & 0 & 0.18 & 0.03 & 0.79 & $X$ & $A 2$ \\
\hline & Sun [3] & 0.090 & 0.377 & 0.102 & 0.431 & $A 2$ & $A 2$ \\
\hline & $\operatorname{Li}[4]$ & 0.1975 & 0.6145 & 0.1880 & & $A 2$ & $A 2$ \\
\hline & Murphy [5] & 0.1543 & 0.7469 & 0.0988 & & $A 2$ & $A 2$ \\
\hline & Deng [6] & 0.1543 & 0.7469 & 0.0988 & & $A 2$ & $A 2$ \\
\hline & Proposed & 0.0267 & 0.9340 & 0.0393 & & $A 2$ & $A 2$ \\
\hline \multirow{7}{*}{$E 1, E 2, E 3$} & D-S [1] & 0 & 0.6316 & 0.3684 & & $A 2$ & $A 2$ \\
\hline & Yager [2] & 0 & 0.018 & 0.0105 & 0.9715 & $X$ & $A 2$ \\
\hline & Sun [3] & 0.175 & 0.218 & 0.135 & 0.472 & $X$ & $A 2$ \\
\hline & $\operatorname{Li}[4]$ & 0.3400 & 0.4066 & 0.2534 & & $A 2$ & $A 2$ \\
\hline & Murphy [5] & 0.3500 & 0.5224 & 0.1276 & & $A 2$ & $A 2$ \\
\hline & Deng [6] & 0.5816 & 0.2439 & 0.1745 & & $A 1$ & $A 2$ \\
\hline & Proposed & 0.0155 & 0.9682 & 0.0163 & & $A 2$ & $A 2$ \\
\hline \multirow{7}{*}{$E 1, E 2, E 3, E 4$} & D-S [1] & 0 & 0.3288 & 0.6712 & & $A 3$ & $A 2$ \\
\hline & Yager [2] & 0 & 0.0018 & 0.00368 & 0.99452 & $X$ & $A 2$ \\
\hline & Sun [3] & 0.194 & 0.160 & 0.137 & 0.509 & $X$ & $A 2$ \\
\hline & $\operatorname{Li}[4]$ & 0.3978 & 0.3250 & 0.2772 & & $A 1$ & $A 2$ \\
\hline & Murphy [5] & 0.6027 & 0.2627 & 0.1346 & & $A 1$ & $A 2$ \\
\hline & Deng [6] & 0.8060 & 00482 & 0.1458 & & $A 1$ & $A 2$ \\
\hline & Proposed & 0.0120 & 0.9795 & 0.0086 & & $A 2$ & $A 2$ \\
\hline \multirow{7}{*}{$E 1, E 2, E 3, E 4, E 5$} & D-S [1] & 0 & 0.1288 & 0.8772 & & $A 3$ & $A 2$ \\
\hline & Yager [2] & 0 & 0.00018 & 0.00129 & 0.99853 & $X$ & $A 2$ \\
\hline & Sun [3] & 0.211 & 0.138 & 0.144 & 0.507 & $X$ & $A 2$ \\
\hline & $\operatorname{Li}[4]$ & 0.4294 & 0.2798 & 0.2909 & & $A 1$ & $A 2$ \\
\hline & Murphy [5] & 0.7958 & 0.0932 & 0.1110 & & $A 1$ & $A 2$ \\
\hline & Deng [6] & 0.8909 & 0.0086 & 0.1005 & & $A 1$ & $A 2$ \\
\hline & Proposed & 0.0114 & 0.9831 & 0.0055 & & $A 2$ & $A 2$ \\
\hline
\end{tabular}


In addition to the method in this paper being able to effectively solve the "Zadeh Paradox" problem [20] and help decision makers make effective decisions, two other following analyses can be got from Table 4:

Conclusion 11: "Not being contrary to intuitive feeling" is still not consistent with "not being contrary to the facts". From the simulation result, when there are only two pieces of evidence $E_{1}$ and $E_{2}$ in the system, all the methods succeed to correctly identify the true target except Yager [2]. When the evidence $E_{3}$ is added, except for Yager [2], Sun [3] and Deng [6], the other methods can still identify the real target and have certain anti-interference ability. When adding evidence $E_{4}$, only the proposed method can identify the true target. When the evidence $E_{5}$ is added at the end, only the proposed method can still identify the true target. However, the remaining methods not only cannot identify the real target, but they also decrease the support for the real target $A 2$. This is because when bad evidence is gradually increasing, the ability of the system to resist interference becomes worse and worse, which makes it increasingly difficult to identify the true target just based on newly collected evidence. As bad evidence gradually increases, the results of this article are increasingly unsatisfied with intuition but not against facts, and can still identify true targets, while the results of other methods are more and more satisfying with intuition, but increasingly against facts or even identify wrong targets.

Conclusion 12: With the increase of bad evidence, the proposed method can still identify the real target, while the performance of other methods gradually deteriorates and some methods even start to identify the wrong target. Therefore, the proposed method has a strong anti-interference ability. The reason is that the proposed model distinguishes newly collected evidence in the second stage according to the historical data in the first stage, which can effectively avoid the interference of bad evidence and shows good anti-interference performance.

\subsection{Verification of Commutative Law and Associative Law}

In order to verify that the method proposed in this paper satisfies the commutative law and associative law, the evidences $E_{1}, E_{2}$, and $E_{3}$ are selected and combined in different orders. In a specific period, three different sensors collected three different evidences $E_{1}, E_{2}$ and $E_{3}$. According to the sequence of evidence collection, there are six orders: $(E 1 \oplus E 2) \oplus E 3 ;(E 2 \bigoplus E 1) \oplus E 3 ;(E 1 \oplus E 3)$ $\bigoplus E 2 ;(E 3 \bigoplus E 1) \oplus E 2 ;(E 2 \bigoplus E 3) \bigoplus E 1 ;(E 3 \oplus E 2) \bigoplus E 1$. In the formula, $\bigoplus$ means combination of evidence by the method proposed in the paper. The evidence before $\bigoplus$ is collected before the evidence after $\bigoplus$. For example, $(E 2 \bigoplus E 3) \bigoplus E 1$ indicates that the evidence $E 2$ is collected first, followed by E3, and finally E1. The calculation results in each order are shown in Table 5.

The following three conclusions can be drawn from Table 5:

Conclusion 13: In each case, $E 1 \bigoplus E 2=E 2 \bigoplus E 1, E 1 \oplus E 3=E 3 \bigoplus E 1, E 2 \bigoplus E 3=E 3 \bigoplus E 2$. So the method proposed in the paper satisfies the commutative law.

Conclusion 14: In each case, $(E 1 \bigoplus E 2) \bigoplus E 3=(E 2 \bigoplus E 1) \bigoplus E 3=(E 1 \bigoplus E 3) \bigoplus E 2=(E 3 \bigoplus$ $E 1) \oplus E 2=(E 2 \bigoplus E 3) \oplus E 1=(E 3 \bigoplus E 2) \oplus E 1$. So the method proposed in the paper satisfies the associative law.

Conclusion 15: The results of the method proposed in this paper do not depend on the order in which the outliers occur because the proposed method satisfies the commutative law and associative law. 
Table 5. Simulation results in different orders by the method proposed in the paper.

\begin{tabular}{|c|c|c|c|c|c|}
\hline Cases & Order & m(A1) & $\mathrm{m}(\mathrm{A} 2)$ & m(A3) & $\mathrm{m}(\mathrm{X})$ \\
\hline \multirow{12}{*}{$\begin{array}{c}\text { First stage in cases where } \\
\text { good/bad evidence } \\
\text { predominates }\end{array}$} & $E 1 \bigoplus E 2$ & 0.1543 & 0.7469 & 0.0988 & \\
\hline & $(E 1 \oplus E 2) \oplus E 3$ & 0.3500 & 0.5224 & 0.1276 & \\
\hline & $E 2 \bigoplus E 1$ & 0.1543 & 0.7469 & 0.0988 & \\
\hline & $(E 2 \bigoplus E 1) \bigoplus E 3$ & 0.3500 & 0.5224 & 0.1276 & \\
\hline & $E 1 \bigoplus E 3$ & 0.6827 & 0.0577 & 0.2616 & \\
\hline & $(E 1 \oplus E 3) \oplus E 2$ & 0.3500 & 0.5224 & 0.1276 & \\
\hline & $E 3 \bigoplus E 1$ & 0.6827 & 0.0577 & 0.2616 & \\
\hline & $(E 3 \bigoplus E 1) \bigoplus E 2$ & 0.3500 & 0.5224 & 0.1276 & \\
\hline & $E 2 \bigoplus E 3$ & 0.2010 & 0.6645 & 0.1346 & \\
\hline & $(E 2 \bigoplus E 3) \bigoplus E 1$ & 0.3500 & 0.5224 & 0.1276 & \\
\hline & $E 3 \bigoplus E 2$ & 0.2010 & 0.6645 & 0.1346 & \\
\hline & $(E 3 \bigoplus E 2) \bigoplus E 1$ & 0.3500 & 0.5224 & 0.1276 & \\
\hline \multirow{12}{*}{$\begin{array}{l}\text { Second stage in cases where } \\
\text { good evidence predominates }\end{array}$} & $E 1 \bigoplus E 2$ & 0.6579 & 0.1053 & 0.2368 & \\
\hline & $(E 1 \bigoplus E 2) \bigoplus E 3$ & 0.7934 & 0.0182 & 0.1884 & \\
\hline & $E 2 \bigoplus E 1$ & 0.6579 & 0.1053 & 0.2368 & \\
\hline & $(E 2 \bigoplus E 1) \oplus E 3$ & 0.7934 & 0.0182 & 0.1884 & \\
\hline & $E 1 \oplus E 3$ & 0.6829 & 0.0522 & 0.2619 & \\
\hline & $(E 1 \oplus E 3) \oplus E 2$ & 0.7934 & 0.0182 & 0.1884 & \\
\hline & $E 3 \bigoplus E 1$ & 0.6829 & 0.0522 & 0.2619 & \\
\hline & $(E 3 \bigoplus E 1) \oplus E 2$ & 0.7934 & 0.0182 & 0.1884 & \\
\hline & $E 2 \bigoplus E 3$ & 0.6954 & 0.0230 & 0.2816 & \\
\hline & $(E 2 \bigoplus E 3) \oplus E 1$ & 0.7934 & 0.0182 & 0.1884 & \\
\hline & $E 3 \bigoplus E 2$ & 0.6954 & 0.0230 & 0.2816 & \\
\hline & $(E 3 \bigoplus E 2) \bigoplus E 1$ & 0.7934 & 0.0182 & 0.1884 & \\
\hline \multirow{12}{*}{$\begin{array}{l}\text { Second stage in cases where } \\
\text { bad evidence predominates }\end{array}$} & $E 1 \bigoplus E 2$ & 0.0267 & 0.9340 & 0.0393 & \\
\hline & $(E 1 \oplus E 2) \oplus E 3$ & 0.0155 & 0.9682 & 0.0163 & \\
\hline & $E 2 \bigoplus E 1$ & 0.0267 & 0.9340 & 0.0393 & \\
\hline & $(E 2 \bigoplus E 1) \oplus E 3$ & 0.0155 & 0.9682 & 0.0163 & \\
\hline & $E 1 \oplus E 3$ & 0.6751 & 0.0717 & 0.2532 & \\
\hline & $(E 1 \bigoplus E 3) \bigoplus E 2$ & 0.0155 & 0.9682 & 0.0163 & \\
\hline & $E 3 \bigoplus E 1$ & 0.6751 & 0.0717 & 0.2532 & \\
\hline & $(E 3 \bigoplus E 1) \bigoplus E 2$ & 0.0155 & 0.9682 & 0.0163 & \\
\hline & $E 2 \bigoplus E 3$ & 0.0081 & 0.9648 & 0.0271 & \\
\hline & $(E 2 \bigoplus E 3) \oplus E 1$ & 0.0155 & 0.9682 & 0.0163 & \\
\hline & $E 3 \bigoplus E 2$ & 0.0081 & 0.9648 & 0.0271 & \\
\hline & $(E 3 \bigoplus E 2) \bigoplus E 1$ & 0.0155 & 0.9682 & 0.0163 & \\
\hline
\end{tabular}

\section{Conclusions}

In summary, the paper adopts the credibility calculation method based on historical data to weight the average of the evidence to be combined, and improve the decision rules to solve the problem that the system cannot make decisions when there are two or more propositions with the maximum support in the combination result. The method proposed in the paper can not only effectively eliminate the "Zadeh paradox" problem caused by the conflict of evidence, but also has strong capability of anti-interference, showing good performance in the case of historical data and no historical data. Moreover, the method has low calculation complexity and fast convergence speed. What's more, it satisfies the commutative law and associative law, which can meet the needs of combination of time-domain evidence. When verifying the validity of the method, this article proposes that the principle of "not being contrary to the facts" should be better than that of "not being contrary to intuitive feeling". Therefore, it has strong application value in the fields of pattern recognition, fault diagnosis, epidemic situation prediction, decision-making assistance and so on.

Author Contributions: Conceptualization, H.W.; methodology, S.Y.; validation, S.Y.; writing—original draft, S.Y.; writing-review and editing, H.W.; funding acquisition, H.W. All authors have read and agreed to the published version of the manuscript.

Funding: This research was supported by National Natural Science Foundation of China, grant number 71962004. 
Conflicts of Interest: The authors declare no conflict of interest.

\section{References}

1. Shafer, G. A Mathematical Theory of Evidence; Princeton University Press: Princeton, NJ, USA, 1976.

2. Yager Ronald, R. On the Dempster-Shafer framework and new combination rules. J. Inf. Sci. 1987, 41, 93-137. [CrossRef]

3. Quan, S.; Xiuqing, Y.; Weikang, G. A New Combination Rules of Evidence Theory. J. Acta Electron. Sin. 2000, 28, 117-119.

4. Bicheng, L.; Bo, W.; Jun, W.; Yuqi, H.; Zhigang, G. Efficient combination rule of evidence theory. J. Data Acquis. Process. 2002, 17, 237-240.

5. Kuenz, M.C. Combining belief functions when evidence conflicts. J. Decis. Support Syst. 2000, 29, 1-9.

6. Yong, D.; Wenkang, S.; Zhenfu, Z. Efficient combination approach of conflict evidence. J. Infrared Millim. Waves 2004, 23, 27-32.

7. Yao, X.; Li, S.; Hu, J. Improving Rolling Bearing Fault Diagnosis by DS Evidence Theory Based Fusion Model. J. Sens. 2017, 2017, 1-14. [CrossRef]

8. Yao, X.; Li, S.; Zhang, A. Equipment Condition Monitoring and Diagnosis System Based on Evidence Weight. Int. J. Online Eng. 2018, 14, 143-154. [CrossRef]

9. Denœux, T.; Sriboonchitta, S.; Kanjanatarakul, O. Evidential clustering of large dissimilarity data. Knowl. Based Syst. 2016, 106, 179-195. [CrossRef]

10. Kang, J.; Gu, Y.B.; Li, Y.B. Multi-sensor information fusion algorithm based on DS evidence theory. J. Chin. Inert. Technol. 2012, 20, 670-673.

11. Smets, P. The combination of evidence in the transferable belief model. IEEE Trans. Pattern Anal. Mach. Intell. 1990, 12, 447-458. [CrossRef]

12. Jousselme, A.L.; Grenier, D.; Bossé, É. A new distance between two bodies of evidence. Inf. Fusion 2001, 2, 91-101. [CrossRef]

13. O'Hagan, M. Aggregating template or rule antecedents in real-time expert systems with fuzzy set logic. In Proceedings of the Twenty-Second Asilomar Conference on Signals, Systems and Computers, Pacific Grove, CA, USA, 31 October-2 November 1988; Volume 2, pp. 681-689.

14. Parikh, C.R.; Pont, M.J.; Jones, N.B. Application of Dempster-Shafer theory in condition monitoring applications: A case study. Pattern Recognit. Lett. 2001, 22, 777-785. [CrossRef]

15. Fan, X.; Zuo, M.J. Fault diagnosis of machines based on D-S evidence theory. Part 1: D-S evidence theory and its improvement. Pattern Recognit. Lett. 2006, 27, 366-376. [CrossRef]

16. Aggarwal, P.; Bhatt, D.; Devabhaktuni, V.; Bhattacharya, P. Dempster Shafer neural network algorithm for land vehicle navigation application. Inf. Sci. 2013, 253, 26-33. [CrossRef]

17. Dong, G.; Kuang, G. Target Recognition via Information Aggregation through Dempster-Shafer's Evidence Theory. IEEE Geosci. Remote Sens. Lett. 2015, 12, 1247-1251. [CrossRef]

18. Swan, M. Blockchain thinking: The brain as a decentralized autonomous corporation. IEEE Technol. Soc. Mag. 2015, 34, 41-52. [CrossRef]

19. Zadeh Lotfi, A. Review of Shafer's: A Mathematical Theory of Evidence. J. AI Mag. 1984, 5, 81-83.

20. Yibing, L.; Jie, C.; Fang, Y.; Dandan, L. The Improvement of DS Evidence Theory and Its Application in IR/MMW Target Recognition. J. Sens. 2015, 2016, 1-15.

21. Sarwar, B.; Karypis, G.; Konstan, J.; Riedl, J. Item-based collaborative filtering recommendation algorithms. In Proceedings of the 10th International World Wide Web Conference, Hong Kong, China, 1-5 May 2001; pp. 285-295.

(C) 2020 by the authors. Licensee MDPI, Basel, Switzerland. This article is an open access article distributed under the terms and conditions of the Creative Commons Attribution (CC BY) license (http://creativecommons.org/licenses/by/4.0/). 